\title{
Monitoring Method for Organic Pollutants in Water and Air
}

\author{
Yingxue Li
}

\author{
School of North China Electric Power University, Baoding, China \\ 602497168@qq.com
}

Keywords: Water pollution; Atmospheric pollution; Environmental pollution monitoring; Chromatogram; Mass spectrum

\begin{abstract}
This paper mainly introduces several methods of monitoring the pollutants in the environment. Using analytical instruments--such as chromatogram, mass spectrum- instead of manual analysis and monitoring, accuracy has been further improved. And the analytical instruments can analyze for the common pollutants in the environment, separate them, and control pollutant source according to the detection results. It can also meet the requirements of environmental analysis and contamination monitoring.
\end{abstract}

\section{对于水体、大气有机污染物的监测方法}

\author{
李预雪 \\ 华北电力大学(保定) 环境科学与工程学院 中国 河北 保定 071000
}

602497168@qq.com

摘要: 该文主要介绍了几种监测环境中污染物的方法。运用分析仪器, 例如色谱仪、质谱仪 等, 代替人工分析监测, 提高了分析的准确度。而且分析仪器能在分析环境中的常见污染物 后, 对这些污染物加以分离, 并根据检出结果对污染物来源进行治理。可满足环境分析和污 物染监测的要求。

关键词: 水体污染; 大气污染; 环境; 污染监测; 色谱; 质谱

\section{1. 引言}

随着经济发展和社会工业化程度的加剧, 大量的有害气体、污水排放到环境中, 都造成了严 重的污染, 从而对人们的日常生活造成了非常严重的影响。据资料统计显示 (水利部门对全国 约 700 条大中河流近 100000 千米河长的检测)，我国现有河流近一半的河段受到污染，10\% 的河流长期污染严重。同时，在过去的几年时间，雾䨪已经侵袭了我国的多座大中型城市。 在这些污染之中, 有机污染占有很大比例, 目前也已成为国际环保工作者研究的热点。对于 环境中的有机污染, 我们必须引起高度重视, 对其进行实时监测, 制定出有效的应对方法, 对有机污染加以控制和治理。在水体、大气环境中, 对有机污染物的分析检测, 是一项十分 复杂的高技术工作。要求仪器具有较高的灵敏度、精密度、准确度。随着当代科学技术的发 展, 攻克诸多技术, 提供了大量的高精度先进仪器, 解决了很多难题。在具有丰富理论和实 践经验的科技人员的操作下, 配合高灵敏度先进仪器的配合使用, 则可对水体、大气环境中 的有机污染物进行测定和监测。

本文对环境中有机化学污染物分析监测的几种主要设备及分析监测技术作简要介绍。

\section{2. 色谱分析法监测}

2.1. 色谱法简介

在化学研究和化工生产中, 色谱法的应用十分广泛。色谱法是一种经典的分离分析方法, 将 待测样品提取、净化后, 可用色谱对其进行分析。色谱按其相态可分为两类, 其中气态为流 
动相的色谱称为气相色谱, 液态为流动相的色谱称液相色谱, 两种色谱都能对污染物进行有效 的检测。下面介绍几种环境分析中常见的色谱方法。

2.2. 常见的几种色谱

2.2.1 气相色谱法

根据固定相状态的差异, 气相色谱又可分为气一固色谱和气一液色谱。气一固色谱是用多孔性固 体为固定相, 针对一些永久性的气体和低沸点的化合物进行分离。气一液色谱多用高沸点的有 机化合物涂渍在惰性载体上作为固定相。具有热稳定性好的化合物, 一般要求在 $480^{\circ} \mathrm{C}$ 以下 且具有 $1500 \mathrm{~Pa}-10000 \mathrm{~Pa}$ 的蒸汽压, 都可用气液色谱分离。

2.2 .2 液相色谱法

液相色谱具有高效、高速、高压的特点, 并且操作简单, 具有极高的灵敏度, 所以使用高效 液相色谱的方法分析污染物有很明显的优势。并且其在有机化合物分析领域应用广，约能鉴 别区分 $80 \%$ 的有机物。

2.2 .3 毛细管色谱

毛细管色谱柱分析法, 是一种高效快捷的分析监测方法。其填料受到的阻力小, 所以气体能 很快通过色谱柱, 很大程度上消除了分析待测组分的浴流扩散所造成的分离不彻底等影响。 其固定液直接涂在管壁上, 增大了色谱柱内壁面积。并且毛细管色谱柱柱效极高, 对环境的 分析监测十分有利。

2.3. 色谱法识图

色谱定性分析的目的是确定每个色谱峰所代表的组份。对色谱峰进行篎选得到监测需要的峰 后，对其剩余组份进行分析，每个组份即代表环境中的一中污染物。

2.4. 色谱法优点与存在问题

色谱分析法分离效率高, 灵敏度高, 速度较快。但与其它仪器分析方法相比, 色谱法的定性 分析能力较弱, 但联用技术的出现使色谱在定性分析方面十分有效, 如 ICP-串联色谱, 色谱 一串联质谱等。对于复杂混合物的分析, 在选择合适的色谱后, 还需要根据试样的质量、物化 性质等因素选取与之联用仪器。根据表一所给数据, 可按照样品质量选择连用条件。

表 1 联用色谱的部分仪器分析方法需要的试样质量要求

\begin{tabular}{ll}
\hline 方法 & 质量 $\mathrm{m} / \mathrm{mg}$ \\
\hline 普通核磁共振波谱 & $1-10$ \\
傅里叶变换核磁共振波谱 & $0.1-1$ \\
红外吸收光谱 & $0.01-0.1$ \\
质谱 & 0.001 \\
\hline
\end{tabular}

\section{3. 核磁共振波谱分析法监测}

3. 1. 核磁共振法原理简介

核磁共振法, 是环境分析中一种重要的方法。由于具有磁矩的原子核在高强度磁场作用下, 可吸收适宜频率的电磁辐射, 而不同分子中原子核的化学环境不同, 将会有不同的共振频率, 产生不同的共振谱。核磁共振的信号强弱与被测磁核的天然丰度与旋磁比的立方成正比。

分析监测试样为液体时, 可以直接进行测定。而待测组分是高分子化合物或岩石、矿物等难 溶物质时, 可用固体核磁共振仪测定。但在大多数分析条件以及实验室中, 通常都采取配成 溶液的方法进行测定。选取溶剂时应注意, 溶剂中不应该含有氢离子等干扰离子, 其对样品 的溶解性应当很好且不与样品发生缔合作用。通常情况下，使用二硫化碳、四氯化碳、丙酮 试剂等。 


\section{4. 质谱分析法监测}

4.1. 质谱法原理简介

质谱法, 是用电场和磁场将运动的离子, 按它们的质荷比分离后进行检测的方法。在质谱分 析中, 对于不同的环境污染样品, 根据其理化性质的不同, 以及形态的不同, 选择不同的分 析器进行分析, 可最大程度上获得最佳的分析监测效果。

质谱分析能够根据谱图信息推测出污染物中元素的具体含量, 进行定量分析。质谱分析法同 样也能推测出污染物的分子结构, 对于不同的同分异构体加以区分, 提高了分析的准确性和 目的性。与核磁共振分析法相似，对环境污染分析和污染治理具有十分重要的意义

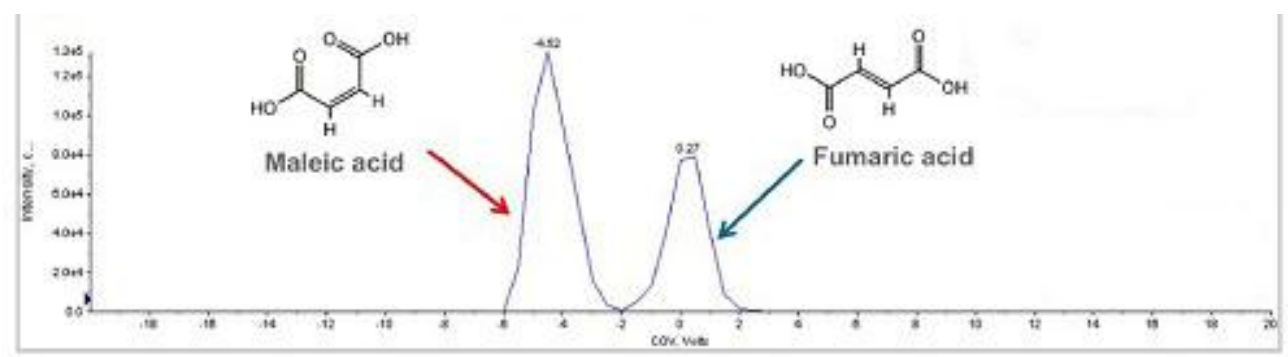

图 1 质谱图中区分顺丁烯二酸（Maleic Acid）和反丁烯二酸（Fumaric Acid）不同的同分

4.2. 常见的质谱质量分析器

异构体

4. 2.1 单聚焦分析器

待测样品离子的比荷很小时, 其在电场中偏转半径也小, 通过磁场的作用可对不同离子进行 选择性分离。设置其它影响因素不变, 改变加速电场电压或磁场强度, 则不同比荷的离子在 不同时间通过狭缝到达检测器, 形成质谱。

4. 2.2 双聚焦分析器

双聚焦质量分析器具有分辨率高的特点。进入离子源的离子仍带有部分能量, 且能量均有差 异。进过电场加速后的离子能量也不相同, 其偏转半径不同, 难以完全聚集。此时, 采用双 聚焦质谱, 分散能量, 则可得到相应结果。

4. 2.3 四极杆分析器

设置其它影响因素不变, 控制了质谱选择的局限性。只有部分离子可通过四极场, 到达检测 器, 选择性大大提高。

4.2. 4 离子阱分析器

其结构简单、灵敏度高。适于小型联用仪器。

4.2 .5 飞行时间分析器

飞行时间分析器采用无聚焦狭缝, 其仪器结构简单, 具有极高的灵敏度, 并且扫描速度快。 在不需要电场、磁场的作用下仍可用于大分子的分析。

4.3. 质谱图识图

在质谱分析的图谱中, 分子离子峰一般是质谱中质量数最大的峰, 根据最大峰和其他峰的位 置，可判断大气、水体中污染物存在的形态和其结构特征。

\section{5. 综合分析法检测}

5. 1. LC-MS 分析方法

即液相色谱-串联质谱方法。在使分析样品得到最佳分离条件并得到最佳电离条件下使用这种 方法。LC 可选择的条件主要有流动相的组成和流速。在此条件下，无机酸、磷酸盐等不挥发 盐类以及表面活性剂由于喷雾雾化和电离因素的影响, 不适合于作流动相。不挥发性的盐会 在离子源内析出结晶, 而表面活性剂会抑制其它化合物电离。因此在 LC-MS 分析方法中常用 的溶剂和缓冲液有水, 甲醇、甲酸、乙酸等。 


\subsection{GC-MS 分析方法}

即气相色谱-串联质谱方法。在 GC-MS 分析方法中, 质谱数据的采集和色谱的分离同时进行。 通常分析中, 其方法可使用毛细管柱作为色谱柱能提高柱效, 便于分离分析。其分析过程中 的电离电压、电子电流、扫描速度、质量范围均可随样品的特性而改变。其分析方法得到的 色谱图中，组分浓度与其色谱峰面积成正比。同时，可采用归一化法、外标法、内标法等不 同方法进行测定。与色谱法定量法相比较而言, GC-MS 分析方法可利用质谱图进行定量分析。 在保证了准确性和高效性的前提下, 增加了质谱图分析, 让分析结果更具有说服力, 同时最 大程度的去除其它杂组份的干扰。

\section{6. 结语}

在进行分析监测之后，根据其结果，确定污染物的来源和分子结构。根据其分子结构得出污 染物种类和物化性质, 采取相应的保护措施应对其可能带来的灾害。根据其来源, 可从根本 上防止污染物的产生。

\section{参考文献}

[1] 武汉大学. 分析化学(下). 第五版. 高等教育出版社, 2007, p.23-45

[2] 朱明华, 胡坪. 仪器分析. 第五版. 高等教育出版社, 2011, p.11-13,34-39

[3] 何世伟. 色谱仪器. 浙江大学出版社, 2012, p.1-9

[4] 高汉宾, 张振芳. 核磁共振原理与试验方法. 武汉大学出版社, 2008, p.51-56

[5] Zelda E, Penton and Fulton G: Kitson, Gas chromatography and mass spectrometry, 科学出版 社, 2013

[6] F.W. Fifield and P.J. Haines, Environment Analytical Chemistry,2004

[7] J.G. Rau, Environmental Impact Analysis Handbook

[8] 汤维敏, 章军. 浅析水监测中有机污染物的检测技术[J]. 工业, 2015(26): 297-297

[9] L Cherta, J Beltran and T Portolés, et al. Multiclass determination of 66 organic micro pollutants in environmental water samples by fast gas chromatography-mass spectrometry [J]. Analytical Bioanalytical Chemistry, 2012, 402(7): 2301-2314

[10]DC Hilton, RS Jones and S Andreas. A method for rapid, non-targeted screening for environmental contaminants in household dust [J]. Journal of Chromatography A, 2010, 1217(44): 6851-6856

\section{References}

[1] WuHan University. Analytical chemistry (ii). Fifth Edition. Higher Education Press, 2007, p. $23-45$

[2] M.H. Zhu, P. Hu: Instrument analysis. Fifth Edition. Higher Education Press, 2011, p.11-13, 34-39

[3] S.W. He: Chromatograph Instrument. Zhejiang University Press, 2012, p.51-56

[4] B.H. Gao, Z.F. Zhang: Principles and test methods of nuclear magnetic resonance. Wuhan university press, 2008

[5] Zelda E, Penton and Fulton G. Kitson: Gas chromatography and mass spectrometry, Science Press, 2013 
[6] F.W. Fifield and P.J. Haines, Environment Analytical Chemistry,2004

[7] J. G. Rau: Environmental Impact Analysis Handbook

[8] M. W. Tang and J. Zhang: Analysis on the detection technology of organic pollutants in water monitoring [J]. Industry, 2015(26): p. 297-297

[9] L Cherta, J Beltran and T Portolés, et al: Multiclass determination of 66 organic micro pollutants in environmental water samples by fast gas chromatography-mass spectrometry [J]. Analytical Bioanalytical Chemistry, 2012, 402(7): p. 2301-2314

[10]DC Hilton, RS Jones and S Andreas. A method for rapid, non-targeted screening for environmental contaminants in household dust [J]. Journal of Chromatography A, 2010, 1217(44): p.6851-6856 